\title{
An Empirical Study on Some Image Enhancement Techniques towards Identification of Chronical Diseases
}

\author{
Sreekanth Puli \\ Research Scholar \\ Dept. of CS\& SE \\ Andhra University, VSKP, India
}

\author{
M. James Stephen, PhD \\ Professor \\ Dept. of CSE \\ WISTM, VSKP, India
}

\author{
P. V. G. D. Prasad Reddy, \\ $\mathrm{PhD}$ \\ *Senior Professor \\ Dept. of CS \&SE \\ Andhra University, VSKP, India
}

\begin{abstract}
Detection and analysis in medical images, image enhancement techniques are one of the most important phases. The main aim of Image enhancement is to produce with suitable image and representation of the transformed image. These enhanced medical images can be used identification of chronical diseases. A lot of work has been done by different researchers and scientists in the field of image enhancement in the recent past. This paper discusses previous research work about the some image enhancement techniques towards identification of chronical diseases and tried to find the most suitable and appropriate image enhancement techniques identification of chronical diseases.
\end{abstract}

\section{Keywords}

Medical image analysis, Image Enhancement, Identification of chronical diseases.

\section{INTRODUCTION}

Chronical diseases are continuing for a long period of time problems that usually can be controlled but not relieve. People living with chronic diseases often must manage daily symptoms that affect their quality of life and health problems and complications that can shorten their life expectancy.

By proper diagnosis of the chronic diseases at the very early stages would certainly help patients in quick recovery. But detecting of these chronic anomaly's with support of auto detection system these gives the motivation towards some image enhancement techniques towards the identification of chronical diseases.

Medical imaging systems often require the application of image enhancement techniques to help physicians in anomaly detection and diagnosis, as well as to improve the quality of images that undergo automated image processing. By using Image Enhancement Techniques For improving the quality of the image and to give better input for processing the image. The enhancement techniques are categorized into two types:

1. Spatial domain methods: In this method, the operation takes place directly on the pixels of the image which in turn leads to contrast enhancement.

2. Frequency domain methods: In this method, the operation takes place on the Fourier transform of the respective image.

Real time solutions are carried out in spatial domain, the same approach taken in this study because it is very simple, easy to interpret and mainly the complexity range is very low. Noise removal from the image plays a vital role and it is also one of the most important tasks in various image enhancement techniques. Filtering is a technique which acts as a tool for removing the noise present in the image. Medical imaging is the technique, process and art of creating visual representations of the interior of a body for clinical analysis and medical intervention. Medical imaging seeks to reveal internal structures hidden by the skin and bones, as well as to diagnose and treat disease. Medical imaging also establishes a database of normal anatomy and physiology to make it possible to identify abnormalities. In this Paper various research outcomes, techniques and models related to medical image enhancements are discussed. An attempt is made to compare various tools and models used in medical image enhancement later at the some better future suggestions are proposed.

\section{RELATED WORK}

This section highlights the related work of various approaches used by different researches in the field of medical image enhancement.

Leonardo Rundo, Andrea Tangherloni [1] presented "MedGA, a novel evolutionary method for image enhancement in medical imaging systems". MedGA, a novel image enhancement method based on Genetic Algorithms was introduced that is able to improve the appearance and the visual quality of images characterized by a bimodal gray level intensity histogram, by strengthening their two underlying sub-distributions. MedGA can be exploited as a preprocessing step for the enhancement of images with a nearly bimodal histogram distribution, to improve the results achieved by downstream image processing techniques.

Mamta Mittal, SumitKaur et al. [2] described deep learningbased approach for brain tumor image segmentation. It includes the concept of Stationary Wavelet Transform (SWT) and new Growing Convolution Neural Network (GCNN). The significant objective of this work is to enhance the accuracy of the conventional system. A comparative analysis with Support Vector Machine (SVM) and Convolution Neural Network $(\mathrm{CNN})$ is carried out in this method.

T. M. Shahriar Sazzad et al. [3] proposed an automated approach where MRI gray-scale images were incorporated for brain tumor detection. This method includes enhancement at the initial stage to minimize gray-scale color variations. Filter operation was used to remove unwanted noises as much as possible to assist better segmentation.

H. Zheng et al.[4] aimed to improve edges of brain MRI by incorporating the gradient information of another contrast high-resolution image. Multi-contrast images are assumed to possess the same gradient direction in a local pattern. In this method a new framework has been proposed to establish a 
relation model of gradient value between different contrast images to restore a high-resolution image from its input lowresolution version. The similarity of image patches is employed to estimate intensity parameters, leading a more accurate reconstructed image. Then, an iterative backprojection filter is applied to the reconstructed image to further increase the image quality.

Navdeep Singh et al. [5] focused on retinal imaging and proposed two techniques, RIHE-RVE (Radiance indicator based histogram equalization for retinal vessel enhancement) and RIHE-RRVE (Radiance indicator based histogram equalization for recursive retinal vessel enhancement) to address the problem of low light radiance. The techniques separate the histogram into sub-histograms at the split value determined by the tuneable parameter, RIHE-RVE recursively performs histogram integration after each split followed by equalization whereas in RIHE-RRVE histogram.split can be done to any level (which is decided by the parameter) followed by equalization and integration.

H. E. M. Abdalla and M. Y. Esmail.et al. [6] described the processes and techniques used in detection brain tumor based on magnetic resonance imaging (MRI) and artificial neural networks (ANN) techniques, Which executed in the different steps of Computer Aided Detection System (CAD) after collected the image data (MRI). First stage is pre-processing and post-processing of MRI images to enhancement it and make it more suitable to analysis then used threshold to segment the MRI images by applied mean gray level method. In the second stage was used the statistical feature analysis to extract features from images. In the third stage the artificial neural networks were designed. The feed forward back propagation neural network with supervised learning were apply as automatic method to classify the images under investigation into tumor or none tumor.

A. Hamad et al.[7] described a strategy to detect the edges of brain tumor from patient's MRI scan images of the brain. This method incorporates with some noise removal functions, followed by improvement features and gain better characteristics of medical images for a right diagnosis using BCET. The result of second stage is subjected to image segmentation by using Fuzzy c-Means (FCM) clustering method. Finally, canny edge detection method is applied to detect the fine edges.

Bora, N. Wasim Rahman and D. JyotiBora [8] described the different segmentation techniques adopted for segmentation of MRI brain Tumour images. Brain images mostly contain noise, in homogeneity and sometimes deviation. Therefore, accurate segmentation of brain images is a very difficult task. The process of segmentation is still challenging due to the diversity of shape, location, and size of the tumour.

Monika Agarwal, Rashima Mahajan [9] made an attempt to integrate range limited and weighted histogram equalization with adaptive gamma correction followed by homomorphic filtering to study the improvement in contrast as well as to preserve the essential details of the image. An image segmentation based on an efficient Otsu's method has been implemented.

L. A. Akter and G. Kwon[10] proposed a new integrated image segmentation method for MRI brain images. A new transformation called Contourlet Transform which is integrated with canny edge detector has been used. For a better segmentation, an enhancement function on the contourlet coefficients has been applied before applying canny edge detector.

S. P. Archa and C. S. Kumar [11] proposed a technique to detect the gliomas by the method of MRI (Magnetic Resonance Imaging). Manual segmentation takes reasonable time since large number of data generated by MRI have to be processed. For accurate segmentation, a novel completely automatic and reliable segmentation based on CNN was proposed. In pre-processing step, intensity normalization, which is not normally used in Convolutional Neural Network $(\mathrm{CNN})$, is applied. Canny edge detection and edge detection using wavelet transform methods were performed on the image. Using PCNN method Image enhancement was done.

R. Lin, Z. Ye, H. Wang .et al.[12] Described recent research efforts and conducts a comprehensive overview of the work on medical big data, especially as related to chronic diseases and health monitoring. It focused on the full cycles of the big data processing, which includes medical big data preprocessing, big data tools and algorithms, big data visualization, and security issues in big data. It also attempts to combine common big data technologies with special medical needs by analyzing in detail existing works of medical big data.

Varuna Shree, Kumar [13] had focused on noise removal technique, extraction of gray-level co-occurrence matrix (GLCM) features, DWT-based brain tumor region growing segmentation to reduce the complexity and improve the performance. This was followed by morphological filtering which removes the noise that can be formed after segmentation. The probabilistic neural network classifier was used to train and test the performance accuracy in the detection of tumor location in brain MRI images.

B. V. Ravindra, N. Sriraam .et al. [14] described use of artificial neural network (ANN) based classification of Chronic kidney disease (CKD) and NCKD. Four attributes, Creatinine, Urea, Sodium and potassium were considered to diagnose the patient suffering from CKD or not. A feed forward back propagation neural network (BPNN) model was employed for classification and the performance of BPNN classifier was evaluated using sensitivity, specificity and classification accuracy.

Monika Agarwal, Rashima Mahajan [15] Proposed an efficient algorithm to cater the limitation of over enhancement with maximum entropy preservation. The proposed algorithm, input image histogram is segmented first based on its valley positions and then weighted distribution is applied to all segmented sub histograms followed by the histogram equalization, gamma correction and homomorphic filtering.

K. Viswanath and Shweta [16] focused on the enhancement of medical brain tumor images in the spatial domain as well as transform domain. In the spatial domain, power law transformation is adopted and in the transform domain, color enhancement by scaling the DCT coefficients has been used. In this technique some parameters are considered which are suitable for medical images and match the different contrast enhancement by scaling techniques with proposed technique using those parameters to obtain appropriate results so that the minor details in the resultant image are enhanced for further analyzation and diagnosis..

Kamil Dimililer, Ahmet Ilhan [17] had focused image processing techniques are applied on MRI images to preserve information details. These techniques are erosion, contrast enhancement and median filtering. The purpose of observe is develop an image processing algorithm for 
brain cancer detection on MRI images. Comparison of back propagation neural networks will be done using original images and reconstructed images on the effect of categorization

Vasupradha Vijay, A.R. Kavitha.et al. [18] had proposed Enhanced Darwinian Particle Swarm Optimization (EDPSO) for automated tumor segmentation which overcomes the drawback of existing Particle Swarm Optimization(PSO). This innovative method consists of four steps. First step is pre-processing, film artifacts and unwanted portions of MRI images are removed using tracking algorithm. Second step involves the process of removing the noises and high frequency component using Gaussian filter. Third step, segmentation is done using Darwinian Particle Swarm Optimization and Fourth step is classification, which is done by Adaptive Neuro Fuzzy Inference System.

H. Kaur and J. Rani [19] Described digital image processing needs efficient MRI image with less noise and improved contrast of image. The main process examined and look at different Histogram based enhancement techniques. Histogram equalization analyze on the bases of Magnetic resonance imaging (MRI) furthermore calculate the metrics parameter of histogram techniques.

K. Bhima and A. Jagan [20] focused on enhancement of accuracy for detection of anomalies in Brain MR Images to improve the accuracy of anomalies detection by merging the results of most conventional Watershed Method and EMGM Method. The application of this method is equipped with the bilateral filter to improve the brain MR image edges for better segmentation.

Saranya G and S. N.Devi [21] used two basic fusion domains, namely spatial domain and transform domain. Principal component analysis (PCA) which is spatial domain technique and discrete cosine transform (DCT), discrete wavelet transform (DWT), stationary wavelet transform (SWT), nonsub sampled contourlet transform (NSCT), and complex contourlet transform (CCT) which are transform domain techniques. Performance metrics are implemented to evaluate and validate the performance of image fusion technique.

Chiao-Min Chen, Chih-Cheng Chen.et al. [22] proposed a hierarchical correlation histogram analysis based on the grayscale distribution degree of pixel intensity by constructing a correlation histogram, that can improves the adaptive contrast enhancement for specific objects. It produces significant results during contrast enhancement pre-processing and facilitates subsequent CAD processes, thereby reducing recognition time and improving accuracy

J.Shin and R. Park [23] proposed a histogram-based localitypreserving contrast enhancement (CE). The method is formulated as an optimization problem to preserve localities of the histogram for performing image contrast Enhancement. The locality-preserving property makes the histogram shape of the enhanced image to be similar to that of the original image

X. Fu, J. Wang, D. Zeng .et al. [24] proposed an empirical approach by using the regularized-histogram equalization (HE) and the discrete cosine transform (DCT) to improve the image quality. First, a new global contrast enhancement method by regularizing the input histogram is introduced. More specifically, this technique uses the sigmoid function and the histogram to generate a distribution function for the input image. The distribution function is then used to produce a new image with improved global contrast by adopting the standard lookup table-based HE technique. Second, the DCT coefficients of the previous contrast improved image are automatically adjusted to further enhance the local details of the image.

S. N. Sulaiman, N. A. Non .et al.[25] described the image segmentation via the clustering method is used to cluster or segment the images into three different regions which represent the white matter (WM), grey matter (GM) and cerebrospinal fluid spaces (CSF), respectively. These regions are significant for the physician or radiographer to analyze and diagnose the disease. The clustering method known as Adaptive Fuzzy K-means (AFKM) is used.

K. R. Mohan and G. Thirugnanam [26] proposed a dualistic sub-image histogram equalization based enhancement and segmentation techniques. Image segmentation plays a vital role in many medical imaging applications by automatically locating the regions of interest. Image segmentation is the most crucial functions in image analysis and processing. Also segmentation results affect all the subsequent processes of image analysis. Manual segmentation of medical image by the radiologist is not only a tedious and time consuming process but also not very accurate.

\section{FINDINGS IN LITERATURESURVEY}

In this area we will talk about different strategies/tools utilized by various authors in their research works. From the above literature review it is concluded that most of the researches used"Histogram Equalization Techniques".

After proper analysis of above-said literature and techniques we can conclude that:

1. The main aim of image enhancement is to improve the visual appearance of an image, or to offer a "better transform representation of the image.

2. One of the most common degradations in medical images is their poor contrast quality and noise.

3. The spatial domain methods lacking the Robustness and imperceptibility.

4. The Morphological Transformation technique can be used to enhance various medical images that can help medical practitioners with better diagnosis and treatment which are invaluable to improve public health.

5. Histogram Equalization Techniques improves the contrast of medical images and can help with better diagnosis.

6. Elastix and Camino is a potential tool in medical image processing.

7. Gimias is another medical image processing tool that provided in framework.

\section{CONCLUSION}

From above survey one can conclude that the Medical images are mostly used as radiographic techniques in diagnosis, clinical studies and treatment planning. By Using Image enhancement techniques in medical images to enhance the contrast and remove the noise to increase image quality. Most of the time histogram equalization techniques used in medical image enhancement to get the better results. Recently Genetic Algorithms and tools has been developed to enhance the medical images. 


\section{REFERENCES}

[1] Leonardo Rundo, Andrea Tangherloni et al "MedGA: A novel evolutionary method for image enhancement in medical imaging systems" Elsevier Expert Systems with Applications, Volume 119,2019, Pages 387-399, ISSN 0957-4174

[2] MamtaMittal, MamtaMittal, SumitKaur et.al "Deep learning based enhanced tumor segmentation approach for MR brain images" Elsevier, Applied Soft Computing, Volume 78, May 2019, Pages 346-354.

[3] T. M. Shahriar Sazzad, K. M. Tanzibul Ahmmed, M. U. Hoque and M. Rahman, "Development of Automated Brain Tumor Identification Using MRI Images," 2019 IEEE International Conference on Electrical, Computer and Communication Engineering (ECCE), Cox'sBazar, Bangladesh, 2019, pp. 1-4.

[4] H. Zheng et al., "Multi-Contrast Brain MRI Image Super-Resolution With Gradient-Guided Edge Enhancement," in IEEE Access, vol. 6, pp. 57856-57867, 2018.

[5] Navdeep Singh, Lakhwinder Kaur, Kuldeep Singh,Histogram equalization techniques for enhancement of low radiance retinal images for early detection of diabetic retinopathy, Elsevier, Engineering Science and Technology, an International Journal, Volume 22, Issue 3,2019,Pages 736-745,ISSN 22150986 .

[6] H. E. M. Abdalla and M. Y. Esmail, "Brain Tumor Detection by using Artificial Neural Network," 2018 IEEE International Conference on Computer, Control, Electrical, and Electronics Engineering (ICCCEEE), Khartoum, 2018, pp. 1-6.

[7] Y. A. Hamad, K. Simonov and M. B. Naeem, "Brain's Tumor Edge Detection on Low Contrast Medical Images," 2018 1st Annual International Conference on Information and Sciences (AICIS), Fallujah, Iraq, 2018, pp. 45-50.

[8] Bora, N. WasimRahman and D. JyotiBora, "Segmentation Techniques for MRI Brain Tumour Images: A Critical Study," 2018 IEEE International Conference on Research in Intelligent and Computing in Engineering (RICE), San Salvador, 2018, pp. 1-5.

[9] Monika Agarwal, Rashima Mahajan, Medical Image Contrast Enhancement using Range Limited Weighted Histogram Equalization, Elsevier, Procedia Computer Science, Volume 125, 2018, Pages 149-156, ISSN 1877 0509 .

[10] L. A. Akter and G. Kwon, "Integration of Contourlet Transform and Canny Edge Detector for Brain Image Segmentation," 2018 IEEE Tenth International Conference on Ubiquitous and Future Networks (ICUFN), Prague, 2018, pp. 798-800.

[11] S. P. Archa and C. S. Kumar, "Segmentation of Brain Tumor in MRI Images Using CNN with Edge Detection," 2018 IEEE International Conference on Emerging Trends and Innovations In Engineering And Technological Research (ICETIETR), Ernakulam, 2018, pp. 1-4.

[12] R. Lin, Z. Ye, H. Wang and B. Wu, "Chronic Diseases and Health Monitoring Big Data: A Survey," in IEEE
Reviews in Biomedical Engineering, vol. 11, pp. 275288, 2018.

[13] Varuna Shree, N. \& Kumar, "Identification and classification of brain tumor MRI images with feature extraction using DWT and probabilistic neural network" Springer Brain Informatics March 2018, Volume 5, Issue 1, pp 23-30.

[14] B. V. Ravindra, N. Sriraam and M. Geetha, "Chronic Kidney Disease Detection Using Back Propagation Neural Network Classifier," 2018 IEEE International Conference on Communication, Computing and Internet of Things (IC3IoT), Chennai, India, 2018, pp. 65-68.

[15] Monika Agarwal, Rashima Mahajan, Medical Images Contrast Enhancement using Quad Weighted Histogram Equalization with Adaptive Gama Correction and Homomorphic Filtering, Elsevier ,Procedia Computer Science, Volume 115, 2017, Pages 509-517, ISSN 1877 0509.

[16] K. Viswanath and Shweta, "Enhancement of brain tumor images," 2017 2nd IEEE International Conference on Recent Trends in Electronics, Information \& Communication Technology (RTEICT), Bangalore, 2017, pp. 1894-1898.

[17] Kamil Dimililer, Ahmet İlhan, Effect of Image Enhancement on MRI Brain Images with Neural Networks, Elsevier , Procedia Computer Science, Volume 102,2016, Pages 39-44, ISSN 1877-0509.

[18] Vasupradha Vijay, A.R. Kavitha, S. Roselene Rebecca, Automated Brain Tumor Segmentation and Detection in MRI Using Enhanced Darwinian Particle Swarm Optimization(EDPSO), Elsevier Procedia Computer Science,Volume 92,2016, Pages 475-480,ISSN 1877 0509 .

[19] H. Kaur and J. Rani, "MRI brain image enhancement using Histogram Equalization techniques," 2016 IEEE International Conference on Wireless Communications, Signal Processing and Networking (WiSPNET), Chennai, 2016, pp. 770-773.

[20] K. Bhima and A. Jagan, "An efficient method for identification of anomalies in brain MR images," 2016 IEEE International Conference on Signal and Information Processing (IConSIP), Vishnupuri, 2016, pp. $1-5$.

[21] Saranya G and S. N. Devi, "Performance evaluation for image fusion technique in medical images using spatial and transform method," 2016 IEEE International Conference on Wireless Communications, Signal Processing and Networking (WiSPNET), Chennai, 2016, pp. 446-450.

[22] Chiao-Min Chen, Chih-Cheng Chen.et al," Automatic Contrast Enhancement of Brain MR Images Using Hierarchical Correlation Histogram Analysis" Springer Journal of Medical and Biological Engineering December 2015, Volume 35, Issue 6, pp 724-734.

[23] J. Shin and R. Park, "Histogram-Based LocalityPreserving Contrast Enhancement," in IEEE Signal Processing Letters, vol. 22, no. 9, pp. 1293-1296, Sept. 2015.

[24] X. Fu, J. Wang, D. Zeng, Y. Huang and X. Ding, "Remote Sensing Image Enhancement Using 
Regularized-Histogram Equalization and DCT," in IEEE Geoscience and Remote Sensing Letters, vol. 12, no. 11, pp. 2301-2305, Nov. 2015.

[25] S. N. Sulaiman, N. A. Non, I. S. Isa and N. Hamzah, "Segmentation of brain MRI image based on clustering algorithm," 2014 IEEE Symposium on Industrial Electronics \& Applications (ISIEA), Kota Kinabalu,
2014, pp. 60-65.

[26] K. R. Mohan and G. Thirugnanam, "A dualistic subimage histogram equalization based enhancement and segmentation techniques for medical images," 2013 IEEE Second International Conference on Image Information Processing (ICIIP-2013), Shimla, 2013, pp. 566-569. 